\title{
Antimicrobial prescribing for urinary tract infections in patients undergoing total hip or knee arthroplasty (THA/TKA)
}

\author{
S Bailin ${ }^{1}$, N Noiseux ${ }^{2}$, J Pottinger ${ }^{3}$, B Johannsson ${ }^{4}$, A Haleem5 ${ }^{5}$ S Johnson ${ }^{6}$, L Herwaldt ${ }^{5 *}$ \\ From 3rd International Conference on Prevention and Infection Control (ICPIC 2015) \\ Geneva, Switzerland. 16-19 June 2015
}

\section{Introduction}

Patients undergoing THA/TKA were screened pre- \& postoperatively with urinalysis (UA) including urine dipstick \& microscopy. We hypothesized that: 1) many patients without evidence of urinary tract infection (UTI) receive antibiotics if clinicians base treatment on UA results alone; 2) a protocol for screening patients for UTI would decrease treatment for UTI without increasing surgical site infection (SSI) rates.

\section{Objectives}

To identify determinants of treatment for UTI among patients undergoing THA or TKA; to assess the effect of implementing a protocol for screening patients for UTI before THA/TKA on antimicrobial use and SSI rates.

\section{Methods}

We conducted a retrospective cohort study of 200 consecutive patients undergoing THA/TKA from 2/21 - 6/30/ 2011 \& a prospective cohort study of 50 patients undergoing these procedures from $5 / 21-7 / 17 / 2012$ to identify factors influencing treatment for UTIs. We conducted a before-after study to assess the outcome of implementing a screening protocol.

\section{Results}

The strongest determinants of pre- or postoperative treatment for UTI were positive leukocyte esterase (LE; $\mathrm{P}<0.0001 ; \mathrm{P}<0.0001)$ and urine white blood cell count $>5(\mathrm{P}=0.01 ; \mathrm{P}=0.01)$. At least $59.7 \%$ of patients treated did not have clinical evidence of UTI. The screening protocol was revised such that all patients with a positive LE or nitrite test have urine cultures. Patients were treated for UTIs if the cultures grow > 100,000 CFU of 1 organism. Subsequently, the number of patients receiving antimicrobial treatment for presumed UTI decreased $80.2 \%$; the SSI rate did not increase.

\section{Conclusion}

A new protocol for diagnosis and treatment of UTIs was associated with a significant decrease in treatment for presumed UTI but the incidence of SSI did not increase. These results suggest that most patients with positive LE do not need treatment for UTI before THA/TKA.

\section{Disclosure of interest}

None declared.

\section{Authors' details}

${ }^{1}$ University of lowa College of Medicine, lowa City, USA. ${ }^{2}$ Orthopaedics, University of lowa College of Medicine, lowa City, USA. ${ }^{3}$ Clinical Quality, Safety, and Process Improvement, University of lowa Hospitals and Clinics, lowa City, USA. ${ }^{4}$ Internal Medicine, Landspitali University Hospital, Reykjavik, Iceland. Internal Medicine, University of lowa College of Medicine, lowa City, USA. ${ }^{6}$ Pharmaceetical Care, University of lowa Hospitals and Clinics, lowa City, USA.

Published: 16 June 2015

doi:10.1186/2047-2994-4-S1-P220

Cite this article as: Bailin et al:: Antimicrobial prescribing for urinary tract infections in patients undergoing total hip or knee arthroplasty (THA/TKA). Antimicrobial Resistance and Infection Control 2015 4(Suppl 1): P220.

${ }^{5}$ Internal Medicine, University of lowa College of Medicine, lowa City, USA Full list of author information is available at the end of the article 